\title{
Design Perspective on the Role of Advanced Bots for Self-Guided Learning
}

\author{
Torsten Reiners \\ Senior Lecturer, School of Information Systems, Curtin Business School, Curtin \\ University, Perth, Western Australia, Australia \\ Lincoln C. Wood \\ Senior Lecturer, Department of Business Information Systems, Faculty of Business \\ and Law, Auckland University of Technology, Auckland, New Zealand \\ Theo J. Bastiaens \\ Chair of Department, Instructional Technology and Media, Fernuni Hagen, Hagen, \\ Germany
}

\begin{abstract}
Virtual worlds are rapidly gaining acceptance in educational settings; with bots play an important role in these environments to help learners. Authentic learning can be significantly supported by bots to help self-guided learning in authentic tasks. In this paper, we investigate what is stopping educators from making more use of bots as a valuable resource. We seek the expertise of six educators, who used educational bots, to find an answer to this question.. While they have 'big plans' for bot use, the current implementations are 'low-level' and restrictive in their application. There is further confusion about appropriate pedagogical models and how to use them effectively as more than 'prompters' or 'extras'. While creation and control technologies are advancing, allowing use of bots as a 'hard technology' to guide learners through routine procedures; there is a lack of resources for automation as intelligence technologies are slower to develop and may require future partnerships with external parties before they are available useable by general educators.
\end{abstract}

Keywords: Virtual Worlds, Bots, Virtual Environments, Authentic Learning, Self-Guided Learning

The use of virtual worlds within education and entertainment has been on the rise during the last decade. While many elements have been considered and studied carefully, one component that appears to have been under appreciated is the humble 'bot', or software robot. These are important components within virtual world environments; however, considering current technologies and approaches, they have been vastly underutilised. New, rapidly developing tools effectively support the emergence of a new class of bots that will be valuable within virtual worlds for educational purposes by embodying greater autonomy and becoming complete 'characters' within the virtual world, but without any human controlling them; i.e., a non-player character (NPC). Wood and Reiners (2013) perceive an increasing scale denoting the intensity of interactivity, differentiating between appropriate investments of effort in creating the right type of bot to suit different pedagogical requirements. Appropriate levels of investment in various elements of virtual worlds will increase the opportunity to incorporate them into other, existing, educational structures and approaches, extending the overall reach of virtual worlds and transforming traditional (i.e., face-to-face), blended and online, learning approaches. This research seeks to understand the opinions of experts and educators on the use of bots and how they can be better utilised, starting with a foundation established by careful design.

This article reviews materials on the use of virtual worlds in education, with a particular emphasis on the value of bots. We then look at the concept of authentic learning and how bots can aid instructional designers. After outlining the methodology we examine both secondary and primary data to understand experts' perspective on bot design and highlight how they may be used in the future.

\section{Literature Review}


This is an electronic Post-Print (i.e., authors' final version, post-refereeing) of an article published as:

Reiners, T., Wood, L. C., \& Bastiaens, T. (2014). Design Perspective on the Role of Advanced Bots for Self-

guided Learning. The International Journal of Technology, Knowledge and Society, 9(4), 187-199.

Three streams of literature are examined. First, how virtual worlds have been used in educational settings. Second, bots and how these contribute to virtual worlds. Third, authentic learning and immersion in education.

Virtual Worlds in Education

We see a future where the bot will evolve and become more a NPC, using artificial intelligence that has advanced adequately, ensuring that the new bots responds capably and in complex manners with learners whether these learners are acting as individuals or as groups. This will improve learner interactivity within virtual worlds, and form an important component within education and gamification frameworks. New technologies are intersecting and colliding in a manner that will enable virtual world developers and instructional designers to propel their use of bots to become more engaging, able to ensnare learners within virtual environments, and promote the immersion of learners to support educational objectives.

Virtual worlds play an increasingly important role in education. They have been used to take the distance out of distance education, increase engagement with online learning students, and blend the new environment with the traditional learning approaches from classroom environment (Dalgarno \& Lee, 2010; Gregory et al., 2010). Wide-spread use of virtual worlds in education has been held back by the lack of authenticity, the clumsiness of the use from the students' perspective, the difficulty of establishing and using the virtual environment from the instructor perspective, and lack of integration within existing learning management system infrastructures. We examine the use of bots in education to understand how their use can be improved. Virtual experiences to support real-world situations have been used in education in several areas such as teacher education (Masters et al, 2012; Gregory et al., 2011), engineering (Bresciani et al., 2010), health sciences (Thompson \& Hagstrom, 2011), and would be valuable in other areas, such as the simulation of hazardous situations for training purposes (Reiners \& Wood, 2013).

\section{Bots}

While there is some research that guides the overall design of bots in educational virtual worlds (Wood \& Reiners, 2013), much research is still required to understand how educators are able to make best use of bots to improve learning outcomes for students. Using bots are particularly important in distance education, where provision of teachers (or even learners) is not always possible (Heller \& Procter, 2010). Yet, commonly applied bot technologies in 3D spaces are capable of interactions with the learner, yet they remain characterised by low fidelity and implementation challenges due to the technical limitations forced upon designers. The evolution from a simple 'performance bot' to an autonomously interacting NPC requires exploration of the motivation and engagement of learners as well as the communication and interaction between actors in the learning environment to identify the challenges that educators face in using bots and how these may be overcome. It can be characterised by five levels in the Stages of Increased Interactivity model (Wood \& Reiners, 2013, pp. 263-265), from lowest to highest: performance, sensory receptor, knowledge, individuality, and autonomy.

Distinctions must also be made as to how bots are implemented and used, using the concepts of 'hard' and 'soft' technologies (Dron et al, 2011). Hard technologies focus on elimination of errors and efficiency; soft technologies emphasise the latent possibilities and potential, through encouraging creativity and new ideas. The 'hardening' of a bot design can occur through communication with the learner or the environment (Paper, 2009). 'Softening' of the bot design can occur through improving the flexibility of reaction of the bot, accommodating multiple and complex interactions, but providing a foil/responsive environment for creative learning experiences. This is most relevant with the fourth ('individuality') and fifth ('autonomy') stages of bot interactivity (Wood \& Reiners, 2013, pp. 263265); yet, careful instructional design means that this will not always be a complex programming tasks (Wood \& Reiners, 2013) and technology exists to enable even non-tech savvy educators to populate virtual worlds with highly interactive bots (Reiners, Wood, \& Bastiaens, under review). Now that the technology is there to enable such implementation, the question changes to how should the design proceed, in light of new paradigms in learning? The variety of bots for virtual worlds is from human supported (puppeteered) bots (TeachLivE n.d.), communication and interaction (Predinger et al. 2011), education (Reiners et al., in press), displaying emotions (Slater and Burden 2009), or guidance in training settings (Hasler et al., 2013; Riedmann et al., under review).

\section{Authentic Learning and Immersion}

Over the past decade there has been increasing technology adoption in universities. However, this has tended to be limited to automating processes and improving course or programme management; 
This is an electronic Post-Print (i.e., authors' final version, post-refereeing) of an article published as:

Reiners, T., Wood, L. C., \& Bastiaens, T. (2014). Design Perspective on the Role of Advanced Bots for Self-

guided Learning. The International Journal of Technology, Knowledge and Society, 9(4), 187-199.

with only little technology-related improvement of the learning process, effective delivery of content, or how assessments can occur. Some researchers have even noted that the increased use of technology is correlated with a deteriorating of teaching practices (Brabazon, 2007). A possible reason for this is that present technology implementation tends to support existing, teacher-centric, delivery of information styled teaching or simplified assessment forms that often merely address basic cognitive levels of regurgitating remembered facts or figures, or demonstrating a rudimentary understanding of concepts (Anderson \& Krathwohl, 2001).

While technology has not changed how teaching occurs, other pedagogical paradigms have emerged to improve learning outcomes for students while engaging with technology; for example authentic learning uses technology to support learning ( Herrington, Reeves, \& Oliver, 2010). In particular, authentic learning has had significant impact by improving student engagement and putting them at the centre of learning (Herrington et al., 2010). A key principle of authentic learning is that learning activities should take place in a near-real-world setting; e.g., the place where the learner would use those skills in the future. While placing students in actual learning spaces may prove impossible, authentic learning can still occur when the learning context accurately reflects how the knowledge would be used in a real environment ( Herrington et al., 2010). This allows similar patterns of problem solving and consideration of important criteria to ensue desired outcomes. Authentic learning tasks, as defined by Herrington et al. (2010), possess ten characteristics including that they should: have realworld relevance; be of suitable complexity and should promote higher-order thinking; enable multiple perspectives and resources to be used; encourage reflection; and result in an outcome or product that has value beyond merely being an artefact for assessment. These features indicate that authentic learning is particularly interesting and valuable in game-type activities but a cursory comparison with many typical learning tasks (e.g., multi-choice questions (MCQ) or short essays on relatively trivial points) indicates that they clearly do not match these requirements.

Assessment is often considered separately to learning activity design; however, even authentic tasks can be rendered value-less if they are coupled with inappropriately matched assessment methods. Especially if many university practices discourage or implicitly limit the use of authentic assessment tasks (Herrington \& Herrington, 2006). However, authentic learning and gamification can be particularly promising in assessment tasks and Wood et al. (2013) present a simple framework that indicates how gamification and authentic learning tasks can be combined in assessment.

\section{Methodology}

An interview schedule was used as a guide to ensure consistency over the interviews and to reach the objectives of the research. ${ }^{1}$ This structuring ensured that the interviews remained focused on the exploration of phenomena of interest. The schedule was used to initiate questions and discussions around the use of bots, allowing the participants to express their own concepts, interpretations, and perceptions about this topic. Thus, the interviews were all slightly different, not following the exact sequence as presented on the schedule. As much as possible, these semi-structured interviews were used to elicit a rich description of the issues surrounding the use of bots. Due to the geographic spread of the participants, in many cases the interview questions were posed by email, enabling them to respond asynchronously over time zones.

Experts were selected based on their publications and work in virtual worlds. They were invited to participate via an email invitation. After six interviews, strong convergence in answers was established, indicating that an acceptable level of saturation had been reached. Data analysis occurred using a data reduction and thematic-coding process. The reduction of data is a process that "sharpens, sorts, focuses, discards, and organizes data in such a way that 'final' conclusions can be drawn and verified" (Miles \& Huberman, 1994, p. 11). The data reduction process condensed the volume of data and this "usually involves some form of coding" (Collis \& Hussey, 2003, p. 253). These codes were

\footnotetext{
${ }^{1}$ The interview schedule included the following questions:

- How have you used Bots in the past?

- How do you intend to use bots in the future?

- How do you use bots to increase user engagement in virtual environments?

- How is communication between bots and users managed in your environments?

- How do you manage the trade-off between extensive programming and value for users?

- How would you LIKE to use bots in the future?

- What challenges prevent you from doing this now?

- How do you think these challenges can be overcome?
} 
This is an electronic Post-Print (i.e., authors' final version, post-refereeing) of an article published as:

Reiners, T., Wood, L. C., \& Bastiaens, T. (2014). Design Perspective on the Role of Advanced Bots for Self-

guided Learning. The International Journal of Technology, Knowledge and Society, 9(4), 187-199.

grouped by theme to highlight the importance of certain themes and topics in a thematic analysis process (Boyatzis, 1998). As further results were obtained they were analysed in light of the emerging trends. We then matched observations and inferences from the literature (secondary data) with the condensed analysis of expert interviews conducted in this research, to manifest a design perspective on key requirements to implement bots in virtual worlds.

\section{Discussion} as follows:

According to secondary data and our expert perspectives, the key finding can be summarised

- bot use is extensive but remains 'low-level', focused on stages $2 \& 3$ of the Stages of Increased Interaction model described above;

- most experts and educators have 'big plans' to expand on bot use;

- to remove confusion about 'how' to use bots in education, experts require pedagogical models that explicitly incorporate bots; and

- lack of technology or expertise to implement bots effectively creates considerable resource constraints.

\section{Complexity of Use}

At present, most educators have made little effective use of bots, whereas the current application of bots tends towards a role as 'extra' (or additional avatar) and 'prompter'. First, extras are used like extras in movies - they fill scenarios with background 'noise' to create an atmosphere in the environment; eliminating the unworldly appearance that empty places inherit when they are empty in contrast to our expectation of liveliness (Wood \& Reiners, 2013). For a real-world example, imagine the creepy sensation of being at the Eifel Tour in Paris during tourism season in the middle of the day and realising no-one else is around you. Regarding the realisation of this type of bots, very little programming or sophistication is required as the bots do not need to interact with human-controlled avatars and can merely follow simple routines. The bots are used as simple type personas or as a 'crowd' of people in the virtual space to create a touch of dynamism. The second common type of used bots is the prompters that issue instructions and guidance to users in a way that is personable and fits within the role of the played scenario. They are intended to interact with users and prompt or nudge them to undertake certain activities. Depending on the complexity and intelligence of their programming, these bots are suited for incorporation into scenarios to either have a simple function of 'meet, greet, and inform' or having a (primitive) supporting role in the narratives to progress the story and keep the user 'on track' (Reiners, Wood, \& Dron, in press).

Here, the use of bots is not to create a sense of interaction with users, but instead to respond to user actions. Thus, a bot may make a statement or take an action in response to what the users' avatars do; however, these user-based-actions are well-defined actions that must trigger the appropriate response from the bot. Pedagogical use therefore relies on heavily scripted and strongly controlled scenarios that allow instructional designers to (correctly) anticipate user responses and actions within the environment. Using bots in this way allows a low-level of complexity in bot incorporation and use, validating the assertion that bot use remains 'stuck' at the stages 2 and 3 of the Stages of Increased Interaction framework (Wood \& Reiners, 2013).

Some educators admit that they still hesitate to make greater use of bots based on the lingering perception that bots and avatars are 'game-oriented' and have less value in educational settings. As a response, there is some refusal to incorporate bots into scenarios and to encourage more bot-based interactions. It is anticipated that this hesitation may linger for several more years before the incoming cohorts have become more familiarised with avatars, bots, and virtual worlds with their game-playing.

\section{Resources}

The lack of resources to create valuable scenarios for users has been widely identified as an issue. Many implementations so far have been limited by resources, forcing many applications to be low-cost by design. Some researchers note that there are difficulties "finding the time" to use bots effectively, indicating that resources are both technical (programming prowess) and man-power (time to undertake programming).

\section{Resources and Creation/Control Technologies}


This is an electronic Post-Print (i.e., authors' final version, post-refereeing) of an article published as:

Reiners, T., Wood, L. C., \& Bastiaens, T. (2014). Design Perspective on the Role of Advanced Bots for Self-

guided Learning. The International Journal of Technology, Knowledge and Society, 9(4), 187-199.

Limitations on resources for creation/control can be overcome by dedicated professionals who invest their own time as a resource into creating scenarios. The emphasis is on the creation and basic control of bots as they interact with users. Further advancements in technology have simplified the creation and control of bots in various scenarios where the bots require a low-level of interaction with users (Reiners et al., under review).

\section{Resources and Automation/Intelligence Technologies}

The limitation on resources for automation/intelligence control requires significantly greater specialist expertise and cannot be as easily overcome. Greater external expertise and resources are required, with few educators willing or able to acquire these. Another example is the 'chat bot'-like Pandora bots, where the technology is ready to be used in virtual worlds and requires almost no technological skill for the 'off the shelf bots'. Yet, specialised scenarios or conversations require scripting of dialogues; including the explosion of possible reactions following the thread of communications over a longer time (Riedmann et al., under review). Authenticity implies that bots are always available and make an adequate reply to a user, and can take the leading role in the communication; driving the conversation in a specific direction to progress on the narrative.

\section{Artificial Intelligence Resources}

The experts believe that it will take several more years before we see greater use of avatars and bots within education and training scenarios. This will be partly driven by professional collaboration with external providers (overcoming barriers to resourcing); improved creation/control technology (making it easier for educators to flexibly use the bots); and improved automation/intelligence technology allowing more adaptive and 'intelligent' bots to be created so that they will play a greater role in "interactive, self-directed 3D learning environments". Finally, many students still find the technology and the use of bots and avatars to be somewhat unnatural; this is a perspective that our experts expect to change with the forthcoming generation of learners - the 'digital natives' (Prensky, 2001) - who have grown up with these technologies (notably, with digital games) and who have greater media literacy.

Other challenges in the implementation simply result from a lack of training how to create bots and use them creatively in the scenarios. This can be overcome in an educational setting by using a project-oriented creation task as a learning activity for students whereby the construction (and maintenance) of the bot is an outcome. This provides students with practical project management skills while delivering functional bots. Buying bots is also a viable option where fairly generic and low-level interaction with bots is required. The purchaser is generally able to configure the bots using provided, built-in scripts, to work towards the required outcomes.

\section{Semantic Analysis and Understanding Actions}

Analysis of text and response to user actions was not identified by the experts. This may be because of the current understanding of how bots are generally used in virtual worlds and the restrictions that are normally given with the available bot technology. The 'technical' level of interest required for this to be a significant issue; where the issue is not apparent as the bots are not being used at an appropriate level. However, the discussions highlight that there is presently no requirement for the bot to understand what a user says, or analyse how a user responds, to effectively achieve many of the desired outcomes. Furthermore, users still assume that artificial intelligence and natural language understanding is not yet on a level that allows practical application; i.e., with respect to accuracy, reliability, and evaluation. That is, a bot might be programmed to react to exact sentences with a matching reply, but it requires interpretation skills in case of alternate words or grammar being used (e.g., differentiating between homonyms and confusing synonyms). The replies to sentences from users that include emotions or sarcasm require a different reply than simple and straight forward questions. Detection of errors and evaluation of the train of argument is critical as misunderstanding terms (e.g. synonyms) can cause problems in communication and either confuse the learner or create an erroneous learning process.

\section{Bots in Education}

Educators are certainly aware of the value that can be created by bots. However, our experts believe that further dissemination, application, and development of advanced bot technology is hindered by both the significant absence of bots in existing pedagogical models and the limited number of ready- 
This is an electronic Post-Print (i.e., authors' final version, post-refereeing) of an article published as:

Reiners, T., Wood, L. C., \& Bastiaens, T. (2014). Design Perspective on the Role of Advanced Bots for Self-

guided Learning. The International Journal of Technology, Knowledge and Society, 9(4), 187-199.

to-use systems incorporating high-level support for bot integration within virtual world learning scenarios. The current technology advances in virtual world's bots relate mainly to chat bots that provide guidance for the user in the virtual space or increasing the dynamics. Nevertheless, even small repertoires of sentences require high investment and do not guarantee full coverage of even a small subset of possible questions by the user. This also ignores the need to implement gestures and emotions to increase authenticity and believability of bots' reaction. While the technology requirements remain an argument for the low use of bots, guidelines about how existing bot technologies can be part of pedagogical models could increase the perception, awareness, and interest in bots. The following list shows some examples how to use bots in different pedagogical models and educational activities.

- Behaviourism: Compared to role-play involving humans, bots are not getting tired of repeating a lesson unlimited times and providing objective formative feedback to reward good and punish bad behaviour.

- Constructivism: Bots act as mentor, observing the learner with respect to achieving or at least heading towards learning objectives; and interfering being initiating a discussion about how to proceed. The support needs to be triggered based on unexpected change of states.

- Action-based learning: Here, the learner is training in authentic scenarios to practice real-life situations. Bots can improve the immersion by participating or being just the background noise for realism.

- Assessment: Assessment situation have to be equal for each learner, a situation that cannot be guaranteed if done by humans as they getting tired or influenced by the learner. Bot on the other hand are not biased and can create the same condition each time; i.e. in distance education.

\section{Future Use of Bots}

What will we see in five or ten years? The experts identified a range of outcomes and benefits.

- Greater realism and immersion, supported by bots. This will improve interactivity, more natural and realistic response, and anticipation of users' actions and communication. This can also encompass the use of bots being used as 'actors' in the creation of machinima (i.e., the creation of video clips based on in-world recordings).

- Creating learning situations that are not possible in real life. Some elements of businesses can be replicated in training (e.g., role playing mistakes in an order); others rely on luck (e.g., weather-based effects); others cannot be replicated (e.g., poor weather coupled with mistakes during an intense week shortened by public holidays). It is also unwise to replicate dangerous situations in real life (e.g., a machine break-down) which can lead to hazards to workers. Virtual environments enable complete control of variables in a way that is safe.

- Routine procedures taught by bots. This will be driven by improvements in creation/control technologies that allow development of a range of tightly scripted, routine, scenarios for training. This is a relatively 'hard technology' use of bots where they are used to prompt users through a series of activities. Using these routinized prompts may also encourage the creation of a 'level-up' effect, allowing incorporation of gradually increasing levels of difficulty assigned to tasks with the instructions and guidance provided by bots being pre-determined (and, thus, easily accomplished).

- Training of 'soft skills' and improved knowledge-creation in students. In contrast to the routine procedures, advances in automation/intelligence technologies will allow more flexible scenarios to be created in educational settings. These will enable effective teaching and assessment of 'soft skills'. They will also enable richer interaction with bots in a way that students will be independently be able to create and develop new responses to situations in experiential learning, improving their knowledge creation in educational settings. Such use of bots in these scenarios represents a very 'soft technology' use.

These design perspectives lead us to postulate three broad categories of technological requirements for bot design in education.

- First, technology that is available today allows creation of simple bots that embody 'performance' and 'sensory receptors'. These can be created with minimal investment of resources, using existing software approaches, and some time/effort by relatively non-specialised instructional designers.

- $\quad$ Second, creation- and control-technologies are required to achieve Level 2. Here, dedicated personnel are able to accomplish relatively complex bot operations, which have the ability to intelligently respond to scenarios and situations in their immediate environment, with complex and carefully established 
This is an electronic Post-Print (i.e., authors' final version, post-refereeing) of an article published as:

Reiners, T., Wood, L. C., \& Bastiaens, T. (2014). Design Perspective on the Role of Advanced Bots for Selfguided Learning. The International Journal of Technology, Knowledge and Society, 9(4), 187-199.

prompts developed by instructional designers. These resources will come from over-time work and additional resourcing from institutions.

- Third, automation/intelligence technologies will be required to achieve the third level of maturity in technology. These will imbue bots with suitable levels of artificial intelligence to allow them to respond appropriately to user actions and interactions. The ability to understand what is said (semantic analysis tools) coupled with the ability to understand, respond, and act appropriately, creates the highest level outcomes - equivalent to an NPC. At present, this type of development can only be taken in an educational setting if a partnership is achieved with an external party that is an expert in these types of technologies. Otherwise, it may be years before such tools are incorporated into the emerging set of control toolsets for bots.

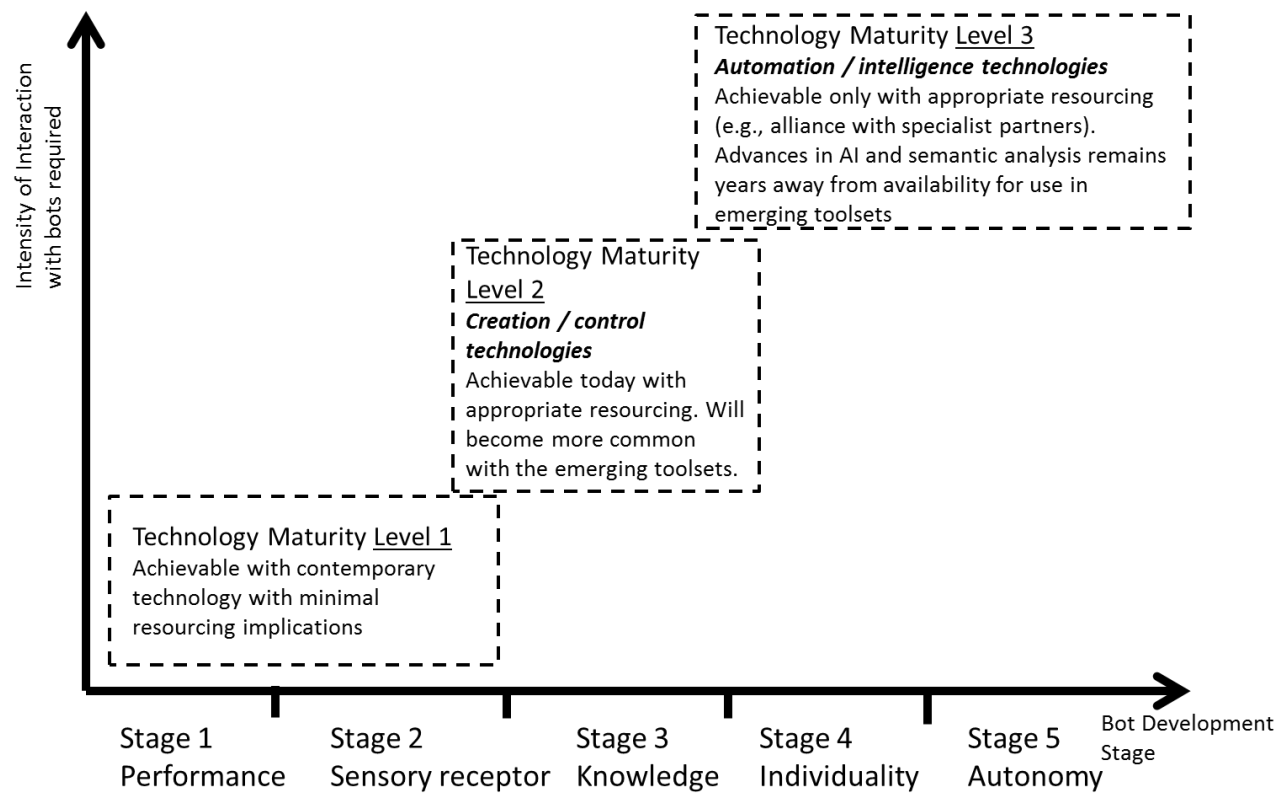

Figure 1 The Bot Interaction and Technology Levels framework, depicting technology requirements for incorporating bots over varying levels of bot interactivity (Based on Figure 11.2 in Wood in Reiners (2013, p. 264)

\section{Conclusion}

We sought to understand, from a design perspective, the use of bots in self-guided learning. There is a body of literature in the developing technologies that support the use of bots (e.g., semantic analysis and artificial intelligence), with strong backup for the value of bots in education (e.g., in authentic learning scenarios and creating authentic assessments). We interviewed experts in education to understand their perspectives, current use, and intended future use of bots. Our findings indicate that there is convergence in the desire to use bots, but varying levels of use. The key barriers that we have identified related to a lack of budget/resources to invest in the projects, and hesitation in adopting new technologies, prejudice against the use of avatars as representing a 'gaming' function. We advance the understanding of the use of bots by presenting the bot interaction and technology levels framework, indicating the progression to creation- and control-technologies required before the attainment of automation and intelligence technologies will allow superior bots to be developed for self-guided learning.

At present, the use of bots in self-guided learning is a 'hard technology' and can only be used effectively for routinized, highly prescribed tasks. Not inconsiderable financial/time resources are required to create even these scenarios; the lack of specialist/technical resources and the lack of advancement in appropriate technologies prevent the attainment of Level 3 bots in self-guided learning. Indications are, that we are five to ten years away from wide-spread adoption of bot technology, resting on these foundations. Future work still needs to be conducted to create effective pedagogical models that explicitly include the use of bots, particularly advanced bots that will leverage developments in automation/intelligence technologies in the coming years. These technologies must be further developed in easily used software tools to enable educators without a technical background to create and implement bots within educational scenarios. 
This is an electronic Post-Print (i.e., authors' final version, post-refereeing) of an article published as:

Reiners, T., Wood, L. C., \& Bastiaens, T. (2014). Design Perspective on the Role of Advanced Bots for Self-

guided Learning. The International Journal of Technology, Knowledge and Society, 9(4), 187-199.

\section{References}

Anderson, L. W., \& Krathwohl, D. R. (2001). A taxonomy for learning, teaching, and assessing: A revision of Bloom's taxonomy of educational objectives (1st ed.). New York, NY: Longman.

Boyatzis, R. E. (1998). Transforming qualitative information: Thematic analysis and code development. Thousand Oaks, CA: Sage Publications.

Brabazon, T. (2007). The University of Google: Education in the post-Information Age. Hampshire: Ashgate Pub Co.

Bresciani, M. J., Morsi, K., Tucker, M., Siprut, M., Stewart, K., \& Duncan, A. (2010). Designing and implementing an assessment plan for a virtual engineering lab. Eludamos: Journal for Computer Game Culture, 4(2), 277-285.

Collis, J., \& Hussey, R. (2003). Business research: A practical guide for undergraduate and postgraduate students (2nd ed.). Basinstoke, Hampshire, UK: Palgrave Macmillan.

Dalgarno, B. and M. J. W. Lee (2010). What are the learning affordances of 3-D virtual environments? British Journal of Educational Technology 40(6): 10-32

Dalgarno, B., Gregory, S., Knox, V., \& Reiners, T. (under review). Practising teaching using virtual classroom role plays. Computers \& Education.

Dron, J., Reiners, T., \& Gregory, S. (2011). Manifestations of hard and soft technologies in immersive spaces. In Proceedings of World Conference on E-Learning in Corporate, Government, Healthcare, and Higher Education (pp. 1895-1904). Chesapeake, VA: AACE.

Gregory, S., Reiners, T., \& Tynan, B. (2010). Alternative Realities: Immersive Learning for and with Students. In H. Song (Ed.), Distance Learning Technology, Current Instruction, and the Future of Education: Applications of Today, Practices of Tomorrow (pp. 245-272). Hershey: IGI Global.

Gregory, S., Dalgarno, B., Campbell, M., Reiners, T., Knox, V., \& Masters, M. (2011). Changing directions through VirtualPREX: Engaging pre-service teachers in virtual professional experience. In G. Williams, N. Brown, B. Pittard, \& B. Cleland (Eds.), Changing Demands, Changing Directions. Proceedings Ascilite (pp. 491-501). Hobart: Univeristy of Tasmania.

Hasler, B. S., Tuchman, P., \& Friedman, D. (2013). Virtual research assistants: Replacing human interviewers by automated avatars in virtual worlds. Computers in Human Behavior, 29(4), 1608-1616. doi:10.1016/j.chb.2013.01.004

Heller, B., \& Procter, M. (2010). Animated pedagogical agents and immersive worlds: two worlds colliding. In G. Velestsianos (Ed.), Emerging Technologies in Distance Education (pp. 301-316). Athabasca University, Edmonton: AU Press.

Herrington, A., \& Herrington, J. (2006). Authentic learning environments in higher education. Hershey, PA: IGI Global.

Herrington, J., Reeves, T. C., \& Oliver, R. (2010). A guide to authentic e-learning. New York, NY: Routledge.

Masters, Y., Gregory, S., Dalgarno, B., Reiners, T., \& Knox, V. (2012). Branching Out through VirtualPREX: Enhancing Teaching in Second Life. In J. Paul, Y. Masters, \& N. Tavares-Jones (Eds.), Utopia and a Garden Party: Experiential Learning in Virtual Worlds, At the Interface: Cutting Edge Research (pp. 57-69). Oxford, United Kingdom: Inter-Disciplinary Press.

Miles, M. B., \& Huberman, A. M. (1994). Qualitative data analysis: An expanded sourcebook (2nd ed.). Thousand Oaks, CA: SAGE Publications Ltd.

Paper, A. W. (2009). 3D Learning and virtual worlds. A. E. i. Action, 1-17.

Predinger, H., S. Ullrich, et al. (2011). "MPML3D: Scripting agents for the 3D internet." IEEE Transactions on Visualization and Computer Graphics 17(5): 655-668.

Prensky, M. (2001). Digital Natives, Digital Immigrants Part 1. On the Horizon, 9(5), 1-6. doi: $10.1108 / 10748120110424816$

Reiners, T., \& Wood, L. C. (2013). Immersive virtual environments to facilitate authentic education in Logistics and supply chain management. In Y. Kats (Ed.), Learning Management Systems and Instructional Design: Metrics, Standards, and Applications (pp. 323-343). Hershey, PA: IGI Global.

Reiners, T., Wood, L. C., \& Bastiaens, T. J. (under review). Mobile application to create interactive learning environments in virtual environments and 3D spaces. The Journal of Learning.

Reiners, T., Gregory, S., \& Knox, V. (in press). Virtual Bots, their Influence on Learning Environments and How They Increase Immersion. In S. Gregory, M. J. W. Lee, B. Dalgarno, \& B. Tynan (Eds.), Virtual Worlds in Open and Distance Education. Athabasca University Press.

Reiners, T., Wood, L. C., \& Dron, J. (in press). From chaos towards sense: A Learner-centric Narrative Virtual Learning Space. In J. Bishop (Ed.), Gamification for Human Factors Integration: Social, Educational, and Psychological Issues. 
This is an electronic Post-Print (i.e., authors' final version, post-refereeing) of an article published as:

Reiners, T., Wood, L. C., \& Bastiaens, T. (2014). Design Perspective on the Role of Advanced Bots for Self-

guided Learning. The International Journal of Technology, Knowledge and Society, 9(4), 187-199.

Riedmann, P., Venable, J., Chang, V., Reiners, T., \& Gütl, C. (under review). RIVALE: A Prototype Realistic Immersive Virtual Agent-based Learning Environment Case Study for Learning Requirements Elicitation Skills. In Proceedings of the AIS SIG-ED IAIM 2013 Conference.

Slater, S. and D. Burden (2009). Emotionally responsive robotic avatars as characters in virtual worlds. First International IEEE Conference on Games and Virtual Worlds for Serious Applications (VSGAMES 2009). Coventry University, Coventry, UK, Curran Associates, IEEExplore: 12-19.

TeachLivE. (n.d.). "TLE TeachLivE." Retrieved 1 September 2013, from http://sreal.ucf.edu/teachlive.

Thompson, C. W. F., \& Hagstrom, F. (2011). Modeling healthcare: Logistics in a virtual world. IEEE Internet Computing, 12(4), 100-104.

Wood, L. C., \& Reiners, T. (2013). Game-based elements to upgrade bots to non-player characters in support of educators. In A. Hebbel-Seeger, T. Reiners, \& D. Schäfer (Eds.), Synthetic Worlds: Emerging Technologies in Education and Economics (pp. 257-277). Berlin: Springer.

Wood, L. C., Teräs, H., Reiners, T., \& Gregory, S. (2013). The role of gamification and game-based learning in authentic assessment within virtual environments. In The Procedings of HERDSA 2013. Presented at the HERDSA 2013, Auckland, New Zealand: HERDSA. 